\title{
Stimulation of the Posterior Parietal Cortex Interferes with Arm Trajectory Adjustments during the Learning of New Dynamics
}

\author{
Valeria Della-Maggiore, ${ }^{1}$ Nicole Malfait, ${ }^{2}$ David J. Ostry, ${ }^{2,3}$ and Tomáš Paus ${ }^{1}$ \\ ${ }^{1}$ Montreal Neurological Institute, McGill University, Montreal, Quebec H3A 2B4, Canada, ${ }^{2}$ Department of Psychology, McGill University, Montreal, Quebec \\ H3A 1B1, Canada, and ${ }^{3}$ Haskins Laboratories, New Haven, Connecticut 06511
}

Substantial neurophysiological evidence points to the posterior parietal cortex (PPC) as playing a key role in the coordinate transformation necessary for visually guided reaching. Our goal was to examine the role of PPC in the context of learning new dynamics of arm movements. We assessed this possibility by stimulating PPC with transcranial magnetic stimulation (TMS) while subjects learned to make reaching movements with their right hand in a velocity-dependent force field. We reasoned that, if PPC is necessary to adjust the trajectory of the arm as it interacts with a novel mechanical system, interfering with the functioning of PPC would impair adaptation. Single pulses of TMS were applied over the left PPC $40 \mathrm{msec}$ after the onset of movement during adaptation. As a control, another group of subjects was stimulated over the visual cortex. During early stages of learning, the magnitude of the error (measured as the deviation of the hand paths) was similar across groups. By the end of the learning period, however, error magnitudes decreased to baseline levels for controls but remained significantly larger for the group stimulated over PPC. Our findings are consistent with a role of PPC in the adjustment of motor commands necessary for adapting to a novel mechanical environment.

Key words: parietal cortex; adaptation; arm dynamics; motor learning; motor control; transcranial magnetic stimulation

\section{Introduction}

To accomplish a reaching movement to a visual target, information about the spatial position of the target and the hand has to be converted into a motor command. This process likely involves a series of transformations from a retinal to a muscle-based coordinate frame (McIntyre et al., 1997; Buneo et al., 2002; BattagliaMayer et al., 2003). Substantial evidence gathered over the past 30 years suggests that specific subregions of the posterior parietal cortex (PPC) are involved in visuomotor transformations underlying visually guided reaching (Kalaska et al., 1983; Goodale and Milner, 1992; Battaglia-Mayer et al., 2000). Neurons located in the medial bank of the intraparietal sulcus (MIP) and adjacent areas $5 \mathrm{v}$ and $\mathrm{V} 6 \mathrm{~A}$, which together form the parietal reach region in the macaque monkey, are active during the planning of reaching and also when the initial motor plan is modified (Snyder et al., 1997, 1998).

Recent studies in humans suggest that, in addition to a role in motor planning, PPC is also necessary for the adjustment of reaching on-line (i.e., once the movement has been initiated). Using transcranial magnetic stimulation (TMS), Desmurget et al. (1999) have shown that the application of single pulses of TMS

\footnotetext{
Received July 14, 2004; revised Sept. 13, 2004; accepted Sept. 15, 2004.

This study was supported by the Natural Sciences and Engineering Research Council of Canada, the Canadian Institutes of Health Research, and the Canadian Foundation for Innovation. We thank Philippe Chouinard and Michael Andres for contributing in setting up the study.

Correspondence should be addressed to Valeria Della-Maggiore, Neuropsychology/Cognitive Neuroscience Unit, Montreal Neurological Institute, Montreal, Quebec H3A 2B4, Canada. E-mail: valeria@bic.mni.mcgill.ca.

DOI:10.1523/JNEUROSCI.2833-04.2004

Copyright $\odot 2004$ Society for Neuroscience $\quad$ 0270-6474/04/249971-06\$15.00/0
}

over PPC at the onset of movement disrupts the adjustment of hand paths when pointing to displaced targets (i.e., targets that shifted location during the saccade) but not to stationary targets. A comparable deficit has been observed in a patient with optic ataxia caused by bilateral damage of the posterior portion of the superior parietal lobes, using a similar experimental paradigm (Pisella et al., 2000). Unlike normal subjects who automatically corrected their hand paths to the displaced target position, the patient was unable to correct the initial trajectory on-line.

The evidence reviewed above suggests that PPC may be crucial when a highly practiced movement needs to be updated but not when it is executed as initially planned. Such updates are expected to take place in certain procedural-learning paradigms in which adaptation to a mechanical perturbation is required, such as in learning new dynamics. When the arm is exposed to an external force while reaching to a target, normal movement kinematics is perturbed, resulting in a deviated arm trajectory. With practice, however, subjects adapt to the distortion by producing the necessary muscle forces to counteract those imposed by the force field (Shadmehr and Mussa-Ivaldi, 1994). The occurrence of aftereffects after removal of the force field suggests that subjects learn to adjust control signals to muscles to compensate for the new dynamics. Here, we tested the possibility that the PPC is necessary for learning new dynamics by applying single pulses of TMS while subjects made reaching movements in a velocitydependent force field. Our results suggest that the posterior parietal cortex participates in the adjustment of motor commands necessary for the adaptation to a novel mechanical environment. 


\section{Materials and Methods \\ Subjects}

Twenty right-handed healthy subjects (10 males and 10 females; $20-35$ years old) participated in this study. Subjects were screened to ensure that none suffered from medical, neurological, or psychiatric disorders and were informed of the experimental procedure before giving written consent. Participants were compensated for their time and inconvenience. The study was approved by the Research Ethics Board of the Montreal Neurological Institute and Hospital.

\section{Experimental setup}

Subjects used the handle of a two-link manipulandum (Interactive Motion, Cambridge, MA) to make $12 \mathrm{~cm}$ point-to-point movements to a single target. At the start position, the hand was aligned with the midline of the body; the initial elbow angle was $90^{\circ}$, and the shoulder angle was $50^{\circ}$ (Fig. $1 \mathrm{~A}$ ). The target, indicated by a lightemitting diode positioned just below the table top, was at $135^{\circ}$ relative to the frontal plane. Shoulder movement was restricted by a harness, and the wrist was braced. The subject's right arm was supported against gravity by an air sled. Participants were trained to produce movements of $500 \pm 50 \mathrm{msec}$ in duration and were instructed to move in a straight line to the target; confirmation of straight-movement intention was assessed through debriefing at the end of the experiment. The robot produced a force field in which the force $(f)$ was a function of the velocity of the hand $(v)$; specifically, $f=B v$, where $B=(0,-23 ; 23,0) \mathrm{N} \cdot \mathrm{sec} \cdot \mathrm{m}^{-1} \cdot$. The field was experienced only during the movement to the target. At the end of each movement, the subjects' hand was brought back to the start position by the robot.

\section{Experimental procedure}

The experiment had two phases separated by $5-10 \mathrm{~min}$. During the first familiarization phase, subjects learned to make movements in the "null field" (that is, while the motors of the robot were turned off). This consisted of six blocks of 30 trials each. During the training phase, a velocitydependent force field was applied at the hand by the manipulandum. This period consisted of five blocks of 30 trials each. The force field was unexpectedly introduced on trial 15 of the first block and removed on trial 16 of the fifth block. The last 15 null-field trials were added to examine the occurrence of aftereffects. Because the effect of eye movements on reaching can be affected by stimulation over certain areas of PPC (Van Donkelaar et al., 2000), subjects were instructed to keep their eyes fixated on the target at all times. Subjects could therefore see their arm only through peripheral vision (no cursor was used to represent hand motion). Resting time ( $<3 \mathrm{~min}$ ) was allowed when needed during each condition.

\section{Data analysis}

Hand positions and forces were sampled at $200 \mathrm{~Hz}$. Position measurements were obtained using encoders in the robotic arm. Forces were measured with a force-torque sensor that was mounted above the handle of the manipulandum. The signals were low-pass Butterworth filtered at $20 \mathrm{~Hz}$ and numerically differentiated. The start and end of movement were defined as $5 \%$ of the maximum tangential velocity, and the data were time normalized. Adaptation was assessed quantitatively in terms of "initial direction error" (Sainburg et al., 1999) defined as the angular distance between the vector from the start position to the target and the vector from the start to the position of the hand at peak of tangential hand velocity. We named this measure initial angular deviation (IAD). We also computed the maximum perpendicular displacement of the hand path from a straight line to the target and obtained qualitatively similar results (data not shown). To assess the difference in the time course of the hand paths across groups, we computed the angular deviation $(\mathrm{AD})$, defined as the angular distance between the vector from the

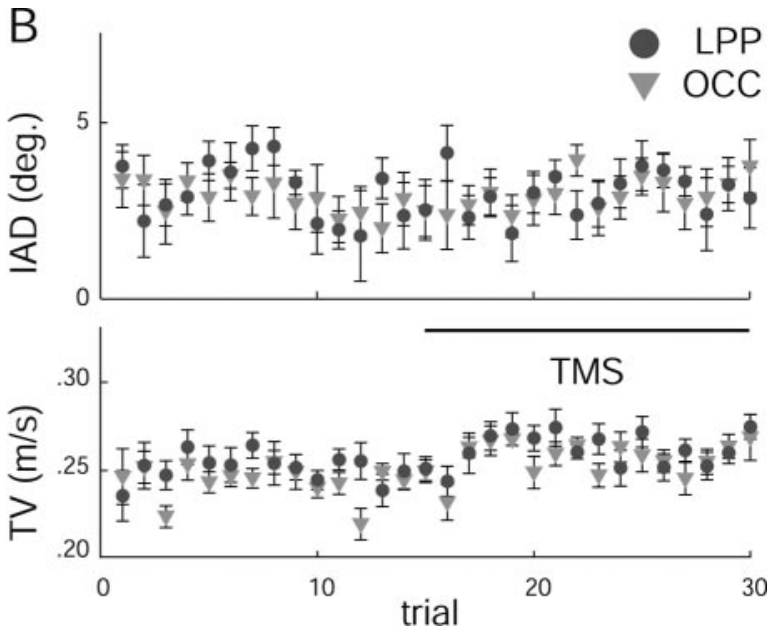

Figure 1. A, Illustrative diagram of the experimental setup. Subjects made center-out planar movements to a visual target using the manipulandum of a robot while their arm was supported by an air sled to reduce friction. $B$, Effect of TMS on the execution fisually guided reaching. Shown are the mean \pm SE corresponding to the 30 trials of the sixth null-field block for the IAD (top) and tangential velocity (TV) (bottom) during the familiarization phase. For practicality, the trials in which TMS was applied figure, as indicated by the horizontal bar.

start to the target and the vector from the start to the position of the hand, at 10 evenly spaced time intervals throughout the movement. Statistical analyses were conducted using repeated-measures ANOVAs, with stimulation site as a between-subjects factor and time as a within-subjects factor. Simple-effect $t$ tests were conducted to break down the interaction when significant.

\section{Transcranial magnetic stimulation}

General procedure. We first determined motor threshold, defined as the intensity of the stimulator necessary to induce a twitch in the first dorsal interosseus muscle of the right hand 5 of 10 times. During this measurement, the TMS coil was placed on the left side of the head, in the portion of the scalp above the hand area of the primary motor cortex. Single pulses were applied using a Magstim 200 magnetic stimulator (Magstim, Whitland, UK) and a figure-of-eight coil. The intensity used for the experiment was $120 \%$ of the motor threshold. Pulses were applied 40 msec after the onset of movement. The timing was based on two previous studies showing a TMS effect of stimulating the posterior parietal area in on-line error detection (Desmurget et al., 1999) and somatosensory discrimination (Oliveri et al., 2000).

To avoid possible confounding effects of interference across adaptation sessions, the effect of TMS on the anatomical target was assessed between groups instead of within group. In half of the subjects (experimental group), the coil was placed over a region corresponding to the left PPC, which we will refer to as left posterior parietal (LPP), and, in the other half (control group), the coil was placed over a region corresponding to the left visual cortex, which we will refer to as occipital (OCC). The stereotaxic coordinates (Talairach and Tournoux, 1988) for LPP $(x=$ $-36, y=-64, z=54$; based on the Montreal Neurological Institute MNI-305 template) were selected based on the results of the following two studies. Using TMS in combination with positron emission tomography, we have shown that this area is functionally connected to the dorsal premotor cortex (Chouinard et al., 2003), which is consistent with the frontal connectivity of its putative homolog (namely, MIP) in the macaque (Burnod et al., 1999; Marconi et al., 2001). We have also shown that TMS applied over this region interferes with the awareness of selfgenerated hand movements, which may require the evaluation of the temporal congruency between peripheral (visual and somatosensory) and central (efference copy) signals (MacDonald and Paus, 2003). The coil was placed over LPP and oriented tangentially and perpendicular to the midline with the handle pointing laterally, so that the induced current flowed in a lateral to medial direction. This orientation was chosen purposely to prevent the occurrence of unspecific TMS effects caused by spread of current toward somatosensory and motor cortices, which lie 
significantly more anterior. The OCC coordinate corresponds to the primary visual cortex, and the stereotaxic coordinates $x=-7, y=-82$, $z=-2$ were extracted from previous neuroimaging studies (Hasnain et al., 1998). For the OCC, the coil was oriented tangentially, with the handle parallel to the midline and pointing down, so that the induced current flowed from the ventral to the dorsal surface of the occipital lobe. With the intensity of stimulation and the coil orientation used in our study, neither interference with processing of visual stimuli nor induction of phosphenes are likely (Amassian et al., 1998). To assess whether TMS interfered with the execution of reaching, stimulation was applied during the last block of the familiarization phase, while subjects performed reaching movements in the null field. Single pulses of TMS were delivered during either the first or the last 15 trials of the block, with the order counterbalanced across subjects. To assess the effect of TMS in learning a new dynamic environment, single pulses of TMS were delivered every force-field trial of the training phase in the force field (i.e., trials 16-30 of block 1 , all trials of blocks 2-4, and trials 1-15 of block 5).

Localization of stimulation sites. After determining the motor threshold, frameless stereotaxy was used to localize the stimulation sites on the subject's scalp. Frameless stereotaxy involved the following steps. First, a high-resolution anatomical image (T1-weighted, $1 \mathrm{~mm}$ thick slice) of the subject's brain was obtained using magnetic resonance imaging (MRI). Second, the two anatomical coordinates corresponding to LPP and OCC were converted from standardized stereotaxic space (Talairach and Tournoux, 1988) into each subject's native coordinate space (Paus, 1999), and the corresponding region was labeled on the MRI. Localization of the scalp positions overlying LPP and OCC was achieved using a three-dimensional infrared optical tracking system [Polaris System (Northern Digital, Bakersfield, CA); Brainsight software (Rogue Research, Montreal, Canada)]. Frameless stereotaxy was also used to track the position of the coil throughout the experiment.

\section{Results}

\section{Effect of single-pulse TMS on the execution of visually} guided reaching

The effect of single-pulse TMS on hand trajectory was first assessed in the null field, during the last block of the first session. Figure $1 B$ shows the mean \pm SE across subjects for the initial angular deviation (top) and the tangential velocity (bottom) of the hand. Observations 1-15 correspond to non-TMS trials, and observations 16-30 correspond to TMS trials. Statistical comparison of IAD for the experimental (LPP) and control (OCC) groups suggests that TMS did not affect the course of arm trajectories when applied during the null field (stimulation site, $F_{(1,18)}=$ $0.031, p=0.86$; time, $F_{(1,18)}=0.456, p=0.51$; TMS, $F_{(1,18)}=$ $0.134, p=0.72$; TMS by stimulation site, $F_{(1,18)}=0.097, p=0.76$; stimulation site by time, $F_{(1,18)}=0.183, p=0.67$; TMS by time, $\left.F_{(1,18)}=3.17, p=0.09\right)$.

Given that the magnitude of the force field imposed during the training phase of the experiment was proportional to the velocity of the hand, an additional repeated-measures ANOVA was conducted on tangential velocity. This allowed us to evaluate the possibility that any TMS effects observed during force-field learning were caused by interfering with the execution of reaching movements. The results revealed that TMS applied under null-field conditions increased the tangential velocity similarly for both stimulation-site groups (TMS, $F_{(1,18)}=18.2, p<0.001$; stimulation site, $F_{(1,18)}=0.005, p=0.95$; TMS by stimulation site, $F_{(1,18)}=0.037, p=0.85$; stimulation site by time, $F_{(28,504)}=$ $0.009, p=0.92)$. The nonspecific facilitative effect of TMS on reaction time has been observed in a variety of tasks, even when the coil is not in contact with the scalp (Terao et al., 1997). Given that single pulses were delivered $40 \mathrm{msec}$ after the onset of movement, it is likely that either the sound or the somatosensory sensation induced at the scalp acted as a facilitative stimulus, speeding up the motor response and, consequently, increasing the
OCCIPITAL

PARIETAL
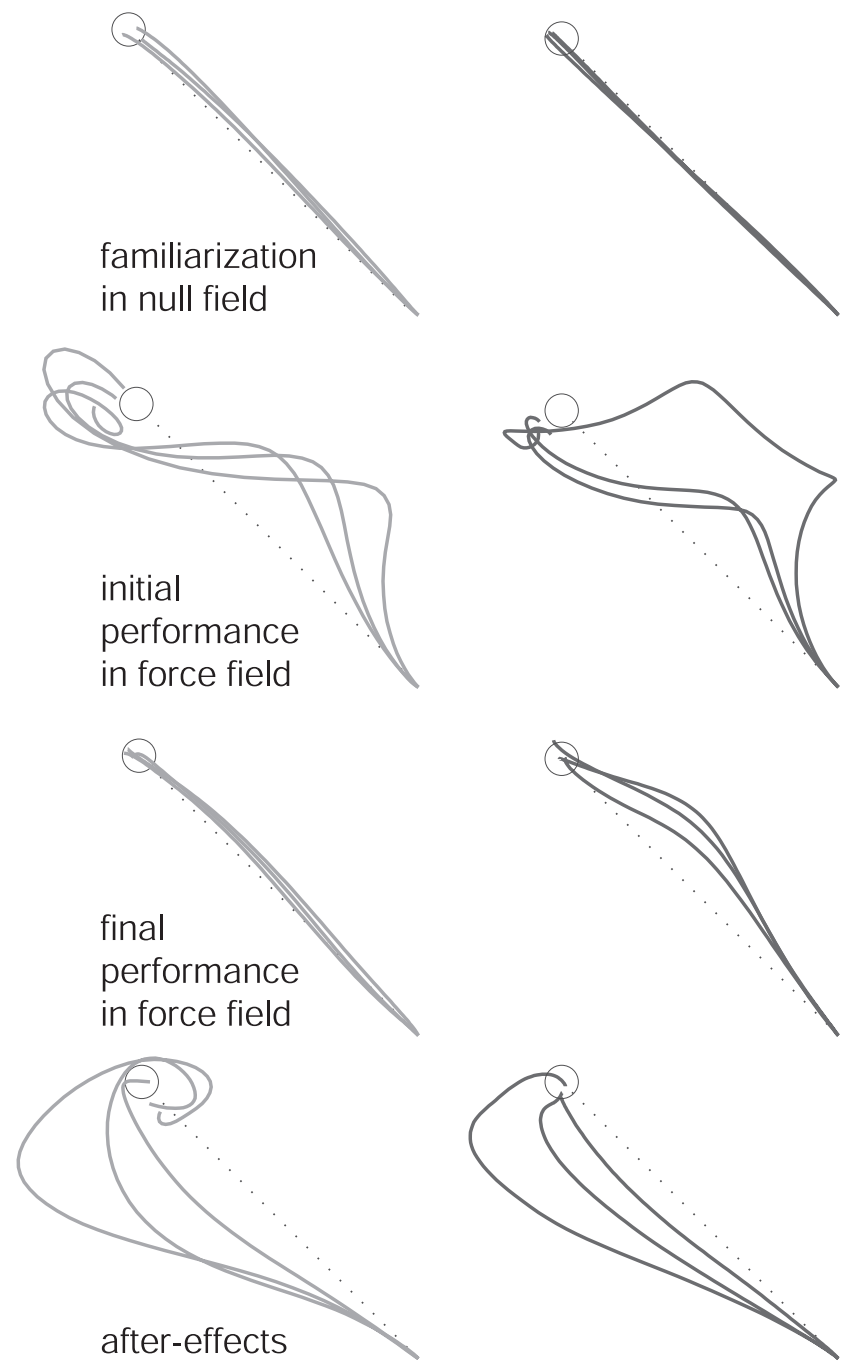

Figure 2. Time course of individual hand paths. Shown are the hand paths for center-out movements in one subject stimulated over LPP and another one stimulated over OCC before, during, and after adaptation to a clockwise force field. Aftereffects represent the resulting arm trajectories after the unexpected removal of the force field during the last block of the training phase.

tangential velocity. Regardless of the possible cause, the fact that hand velocity increased in both groups suggests that this effect was not specific to the site of stimulation and thus could not explain group differences obtained during learning in the force field.

\section{Effect of single-pulse TMS on adaptation to a force field}

The force field was introduced unexpectedly during the first block (trial 16) and removed during the fifth block (trial 16) of the force-field training phase. Figure 2 illustrates the change in the curvature of hand trajectories for an experimental and a control subject throughout the following four different stages of the second session: (1) null field, (2) initial exposure to the force field, (3) final stages in the force field, and (4) after removal of the force field during the fifth block (aftereffects). As observed in previous reports, hand paths during the null field unfolded along a straight line to the target. This was true for both subjects. Both subjects also experienced a consistent deviation in their hand 

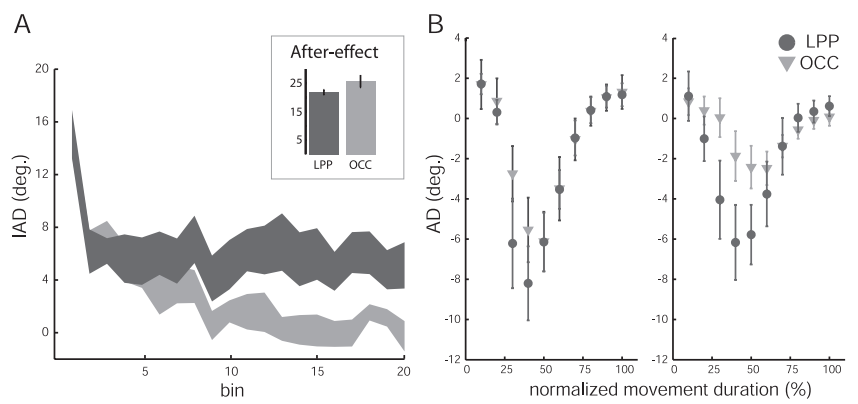

Figure 3. Effect of TMS on adaptation to a new dynamic environment. $A$, Shown are the mean \pm SE of the initial angular deviation as a function of time. The 120 trials of the force field were binned every six trials, yielding a total of 20 bins. The inset shows the mean \pm SE of the $I A D$ corresponding to the first aftereffect trial for both groups. $B$, Shown are the mean $\pm S E$ of the $A D$ as a function of normalized movement duration. $A D$ was computed at 10 evenly spaced intervals over the course of the movement for bin 4 (left) and bin 10 (right) of $A$. Circles, LPP; triangles, $0 \mathrm{CC}$.

trajectories when they first interacted with the force field. These trajectories straightened significantly toward the end of the training period for the subject stimulated over OCC but not as much for the subject stimulated over LPP. Both experimental and control subjects exhibited aftereffects, although their size was larger for the subject stimulated over OCC.

To quantify the effect of TMS on the degree of adaptation, we computed the IAD for all 120 trials in the force field (blocks 1-5). Subsequently, for statistical purposes, we averaged the IAD every six trials, thus obtaining a total of 20 bins. Figure $3 A$ shows the mean \pm SE of IAD for the LPP and OCC groups. Statistical analysis indicated that the effect of TMS on learning a new dynamic environment depended on the site of stimulation (stimulation site, $F_{(1,18)}=3.94, p=0.06$; time, $F_{(19,342)}=18.31, p<0.001$; time by stimulation site, $\left.F_{(19,342)}=3.19, p<0.001\right)$. Although it is clear from the figure that both groups initially learned the task similarly (bins 1-7), the larger deviation observed for the experimental group throughout the second half of the training suggests that stimulating the LPP prevented subjects from fully correcting their hand paths to compensate for the perturbation. Additional examination of the interaction revealed significant differences for bins $8-20$ across groups (bin $8, t=-3.72 ; \mathrm{df}=18 ; p=0.01$ ). The difference in the time course of hand paths across groups is illustrated by Figure $3 B$, which portrays the angular deviation computed at 10 evenly spaced intervals over the course of the movement for bins 4 (left) and 10 (right) of Figure $3 A$. Bin 4 corresponds to trials 19-24, whereas bin 10 corresponds to trials 55-60 of the force field. Symbols represent the mean \pm SE of AD across subjects. As illustrated by Figure $3 B$, no differences in the magnitude of $\mathrm{AD}$ were observed at any point of the arm trajectories for bin 4 of the force field (stimulation site, $F_{(1,18)}=0.419$, $p=0.53$; time by stimulation site interaction, $F_{(9,216)}=0.837$, $p=0.583$ ), but they were evident for bin 10 (stimulation site, $F_{(1,18)}=1.47, p=0.24$; time by stimulation site interaction, $\left.F_{(9,74)}=2.644, p=0.007\right)$.

To determine further whether TMS over LPP affected the learning of new dynamics, group differences in the size of aftereffects measured during block 5 were examined. To assess this possibility, we conducted an a priori one-tailed $t$ test on the first null-field (catch) trial. The analysis, which approached significance at $\alpha=0.06(t=-1.59 ; \mathrm{df}=18 ; p=0.06)$, showed that the size of the aftereffect for the OCC group was larger than that obtained for the LPP group (Fig. $3 A$, inset). The extent of the aftereffect was limited. An overall statistical analysis on all 15 aftereffect trials yielded no significant results (stimulation site, $F_{(1,18)}=0.16, p=0.9$; stimulation site by time, $F_{(14,252)}=1.032$, $p=0.422)$.

\section{Discussion}

In this study, we tested whether interfering with the functioning of the posterior parietal cortex by delivering single pulses of TMS $40 \mathrm{msec}$ after the onset of a reaching movement affected adaptation to a new dynamic environment. Although learning was initially unaffected, TMS significantly interfered with armtrajectory adjustments during later stages of adaptation. The fact that the control group, stimulated over the occipital cortex, exhibited straight hand paths by the end of the experiment indicates that this effect was specific to the stimulation of LPP.

Although TMS was effective in interfering with adjustments of hand trajectories in the force field, it had no specific effect when applied over LPP in the null field. This finding is consistent with recent studies suggesting that $\mathrm{PPC}$ is necessary to generate an error signal when either the course or the plan of a movement needs to be updated but not when executed as originally planned. In one of these studies, Clower et al. (1996) showed that handpath corrections during early stages of adaptation to displaced visual input were associated with increased blood flow in the PPC contralateral to the moving hand. Using a response-switching paradigm based on the presentation of visual cues, Rushworth et al. (2001a) showed that TMS applied over PPC disrupted performance when subjects had to switch the movement from the one initially instructed by a precue but not when the movement remained unaltered. Similar results have been reported using the "unconscious" double-step pointing paradigm, designed to study the adjustment of arm trajectories on-line (Goodale et al., 1986; Pelisson et al., 1986; Prablanc and Martin, 1992). The task consists of making rapid pointing movements to a visual target, the location of which can be modified during the course of the initial saccade. Participants successfully correct their hand paths to the updated target location with latencies as short as $110 \mathrm{msec}$ (Desmurget and Grafton, 2000). Using this paradigm, Desmurget et al. (1999) have demonstrated that single pulses of TMS applied over PPC interfered with pointing movements directed to displaced but not stationary targets. Based on this finding, the authors have proposed that PPC may generate an error signal based on the comparison of the current position of the hand and the target.

Here, we show that manipulating PPC activity in a paradigm in which the perturbation affected the position of the arm, instead of the position of the target, similarly impaired the adjustment of the motor command. Interestingly, the effect was observed despite the fact that the perturbation used in our experimental paradigm was predictable across trials, suggesting that this region of the PPC may contribute to the generation of a corrective motor command regardless of the nature of the task. The region of the parietal cortex targeted in our study, which in humans is functionally connected with the dorsal premotor cortex (Chouinard et al., 2003), has been suggested as a putative homolog of MIP in the macaque (Rushworth et al., 2001b; Andersen and Buneo, 2002). In the monkey, this region receives both proprioceptive and visual inputs (Battaglia-Mayer et al., 2001), as well as information from the premotor cortex. This makes it a suitable neural substrate to compute an error signal using the position of the hand and the target, the former based on central [motor corollary discharge (Sperry, 1950)] and/or peripheral (proprioceptive, visual) input. Alternatively, the position of the hand may have been estimated solely based on propri- 
oceptive and central input. Given that visual feedback was not experimentally manipulated here, we cannot discard this possibility.

Unlike our initial prediction, TMS only interfered with the correction of arm trajectories during later stages of adaptation. This is reflected in the similarity of IAD profiles for the first seven bins (42 trials) and the occurrence of aftereffects in both groups. These results are consistent with those by Thoroughman and Shadmehr (1999) showing that the timing of adjustments to motor commands varies over the course of force-field adaptation. Using the electromyographic response (EMG) of the biceps as the output of the motor command, the authors showed that the EMG peak shifted from $\sim 250 \mathrm{msec}$ during early exposure to the force field to $\sim 70 \mathrm{msec}$ after training. Thus, it is possible that the timing of stimulation chosen in our study ( $40 \mathrm{msec}$ from the onset of movement) was inefficient at interfering with LPP when subjects were first exposed to the force field but became effective later in adaptation, once adjustments to motor commands occurred earlier in the movement.

Alternatively, it is possible that PPC was not involved during early stages of adaptation. Although this option cannot be ruled out by our results, it is inconsistent with the reliable neurophysiological and neuropsychological evidence pointing to the PPC as a necessary structure for accurately directing reaching movements to visual targets (Goodale and Milner, 1992; Andersen and Buneo, 2003). The large directional errors experienced by patients with optic ataxia while reaching or shaping their hand according to the orientation of the object are a compelling illustration of this view (Perenin and Vighetto, 1988; Jakobson et al., 1991; Battaglia-Mayer and Caminiti, 2002; Rossetti et al., 2003). If PPC were necessary for accurate reaching, one would expect it to be active, especially during initial exposure to the force field, when arm trajectories experienced the largest deviations.

An important prediction of our study was that the stimulation of LPP would indirectly interfere with adaptation to a force field by precluding the initiation of corrective motor commands necessary to counteract the force field. The latter process may be mediated through other structure(s), among which the cerebellum is a suitable candidate (Thach et al., 1992; Shadmehr and Holcomb, 1997; Desmurget et al., 2001; Nezafat et al., 2001). A reliable behavioral marker to estimate the degree of learning new dynamics is the occurrence of aftereffects, which reflects the changes in the motor command that are necessary to counteract, in an anticipatory way, the external forces (Shadmehr and Mussa-Ivaldi, 1994). The size of the aftereffects observed in our study suggests that applying TMS over LPP did not prevent the formation of a memory for the new dynamic environment. However, the magnitude obtained for the first null-field trial, the only one that occurred unexpectedly, was marginally larger for the control group, supporting the possibility that TMS may indeed have interfered with learning during later but not early stages of adaptation. The observation that the magnitude of aftereffects increases mostly during early stages of adaptation supports this interpretation (Shadmehr and Mussa-Ivaldi, 1994). Alternatively, it is possible that TMS differentially affected trajectory corrections but spared adaptation. It has been proposed that these mechanisms may rely on parallel errorprocessing systems, with trajectory corrections being processed on-line and adaptation occurring off-line (Pisella et al., 2004). In that case, trajectory adjustments would not be necessary to achieve adaptation. Additional research would be necessary to evaluate these possibilities.

\section{Conclusions}

We have demonstrated that interfering with the functioning of the posterior parietal cortex $40 \mathrm{msec}$ after the onset of a reaching movement impaired adaptation to a velocity-dependent force field. The persistent large errors in hand paths observed for the experimental group together with the timing of stimulation suggest that TMS specifically hindered the adjustment of reaching trajectories. Our results are consistent with a role of PPC in generating an error signal based on the position of the hand and the target. During learning to reach in a new predictable dynamic environment, this error signal would feed back into other brain regions (such as the cerebellum) resulting in the adjustment of descending motor commands and the generation of a memory for the new dynamic environment.

\section{References}

Amassian VE, Cracco RQ, Maccabee PJ, Cracco JB, Rudell AP, Eberle L (1998) Transcranial magnetic stimulation in study of the visual pathway. J Clin Neurophysiol 15:288-304.

Andersen RA, Buneo CA (2002) Intentional maps in posterior parietal cortex. Annu Rev Neurosci 25:189-220.

Andersen RA, Buneo CA (2003) Sensorimotor integration in posterior parietal cortex. Adv Neurol 93:159-177.

Battaglia-Mayer A, Caminiti R (2002) Optic ataxia as a result of the breakdown of the global tuning fields of parietal neurones. Brain 125:225-237.

Battaglia-Mayer A, Ferraina S, Mitsuda T, Marconi B, Genovesio A, Onorati P, Lacquaniti F, Caminiti R (2000) Early coding of reaching in the parietooccipital cortex. J Neurophysiol 83:2374-2391.

Battaglia-Mayer A, Ferraina S, Genovesio A, Marconi B, Squatrito S, Molinari M, Lacquaniti F, Caminiti R (2001) Eye-hand coordination during reaching. II. An analysis of the relationships between visuomanual signals in parietal cortex and parieto-frontal association projections. Cereb Cortex 11:528-544.

Battaglia-Mayer A, Caminiti R, Lacquaniti F, Zago M (2003) Multiple levels of representation of reaching in the parieto-frontal network. Cereb Cortex 13:1009-1022.

Buneo CA, Jarvis MR, Batista AP, Andersen RA (2002) Direct visuomotor transformations for reaching. Nature 416:632-636.

Burnod Y, Baraduc P, Battaglia-Mayer A, Guigon E, Koechlin E, Ferraina S, Lacquaniti F, Caminiti R (1999) Parieto-frontal coding of reaching: an integrated framework. Exp Brain Res 129:325-346.

Chouinard PA, Van Der Werf YD, Leonard G, Paus T (2003) Modulating neural networks with transcranial magnetic stimulation applied over the dorsal premotor and primary motor cortices. J Neurophysiol 90:1071-1083.

Clower DM, Hoffman JM, Votaw JR, Faber TL, Woods RP, Alexander GE (1996) Role of posterior parietal cortex in the recalibration of visually guided reaching. Nature 383:618-621.

Desmurget M, Grafton S (2000) Forward modeling allows feedback control for fast reaching movements. Trends Cogn Sci 4:423-431.

Desmurget M, Epstein CM, Turner RS, Prablanc C, Alexander GE, Grafton ST (1999) Role of the posterior parietal cortex in updating reaching movements to a visual target. Nat Neurosci 2:563-567.

Desmurget M, Grea H, Grethe JS, Prablanc C, Alexander GE, Grafton ST (2001) Functional anatomy of nonvisual feedback loops during reaching: a positron emission tomography study. J Neurosci 21:2919-2928.

Goodale MA, Milner AD (1992) Separate visual pathways for perception and action. Trends Neurosci 15:20-25.

Goodale MA, Pelisson D, Prablanc C (1986) Large adjustments in visually guided reaching do not depend on vision of the hand or perception of target displacement. Nature 320:748-750.

Hasnain MK, Fox PT, Woldorff MG (1998) Intersubject variability of functional areas in the human visual cortex. Hum Brain Mapp 6:301-315.

Jakobson LS, Archibald YM, Carey DP, Goodale MA (1991) A kinematic analysis of reaching and grasping movements in a patient recovering from optic ataxia. Neuropsychologia 29:803-809.

Kalaska JF, Caminiti R, Georgopoulos AP (1983) Cortical mechanisms related to the direction of two-dimensional arm movements: relations in parietal area 5 and comparison with motor cortex. Exp Brain Res 51:247-260. 
MacDonald PA, Paus T (2003) The role of parietal cortex in awareness of self-generated movements: a transcranial magnetic stimulation study. Cereb Cortex 13:962-967.

Marconi B, Genovesio A, Battaglia-Mayer A, Ferraina S, Squatrito S, Molinari M, Lacquaniti F, Caminiti R (2001) Eye-hand coordination during reaching. I. Anatomical relationships between parietal and frontal cortex. Cereb Cortex 11:513-527.

McIntyre J, Stratta F, Lacquaniti F (1997) Viewer-centered frame of reference for pointing to memorized targets in three-dimensional space. J Neurophysiol 78:1601-1618.

Nezafat R, Shadmehr R, Holcomb HH (2001) Long-term adaptation to dynamics of reaching movements: a PET study. Exp Brain Res 140:66-76.

Oliveri M, Caltagirone C, Filippi MM, Traversa R, Cicinelli P, Pasqualetti P, Rossini PM (2000) Paired transcranial magnetic stimulation protocols reveal a pattern of inhibition and facilitation in the human parietal cortex. J Physiol (Lond) 529:461-468.

Paus T (1999) Imaging the brain before, during, and after transcranial magnetic stimulation. Neuropsychologia 37:219-224.

Pelisson D, Prablanc C, Goodale MA, Jeannerod M (1986) Visual control of reaching movements without vision of the limb. II. Evidence of fast unconscious processes correcting the trajectory of the hand to the final position of a double-step stimulus. Exp Brain Res 62:303-311.

Perenin MT, Vighetto A (1988) Optic ataxia: a specific disruption in visuomotor mechanisms. I. Different aspects of the deficit in reaching for objects. Brain 111:643-674.

Pisella L, Grea H, Tilikete C, Vighetto A, Desmurget M, Rode G, Boisson D, Rossetti Y (2000) An "automatic pilot" for the hand in human posterior parietal cortex: toward reinterpreting optic ataxia. Nat Neurosci 3:729-736

Pisella L, Michel C, Gréa H, Tilikete C, Vighetto A, Rossetti Y (2004) Preserved prism adaptation in bilateral optic ataxia: strategic versus adaptive reaction to prisms. Exp Brain Res 156:399-408.

Prablanc C, Martin O (1992) Automatic control during hand reaching at undetected two-dimensional target displacements. J Neurophysiol 67:455-469.

Rossetti Y, Pisella L, Vighetto A (2003) Optic ataxia revisited: visually guided action versus immediate visuomotor control. Exp Brain Res 153:171-179.

Rushworth MF, Ellison A, Walsh V (2001a) Complementary localization and lateralization of orienting and motor attention. Nat Neurosci 4:656-661.

Rushworth MF, Paus T, Sipila PK (2001b) Attention systems and the organization of the human parietal cortex. J Neurosci 21:5262-5271.

Sainburg RL, Ghez C, Kalakanis D (1999) Intersegmental dynamics are controlled by sequential anticipatory, error correction, and postural mechanisms. J Neurophysiol 81:1045-1056.

Shadmehr R, Holcomb HH (1997) Neural correlates of motor memory consolidation. Science 277:821-825.

Shadmehr R, Mussa-Ivaldi FA (1994) Adaptive representation of dynamics during learning of a motor task. J Neurosci 14:3208-3224.

Snyder LH, Batista AP, Andersen RA (1997) Coding of intention in the posterior parietal cortex. Nature 386:167-170.

Snyder LH, Batista AP, Andersen RA (1998) Change in motor plan, without a change in the spatial locus of attention, modulates activity in posterior parietal cortex. J Neurophysiol 79:2814-2819.

Sperry RW (1950) Neural basis of the spontaneous optokinetic response produced by visual inversion. J Comp Physiol Psychol 43:482-489.

Talairach J, Tournoux P (1988) Co-planar stereotaxic atlas of the human brain. Stuttgart, Germany: Thieme.

Terao Y, Ugawa Y, Suzuki M, Sakai K, Hanajima R, Gemba-Shimizu K, Kanazawa I (1997) Shortening of simple reaction time by peripheral electrical and submotor-threshold magnetic cortical stimulation. Exp Brain Res 115:541-545

Thach WT, Goodkin HP, Keating JG (1992) The cerebellum and the adaptive coordination of movement. Annu Rev Neurosci 15:403-442.

Thoroughman KA, Shadmehr R (1999) Electromyographic correlates of learning an internal model of reaching movements. J Neurosci 19:85738588 .

Van Donkelaar P, Lee JH, Drew AS (2000) Transcranial magnetic stimulation disrupts eye-hand interactions in the posterior parietal cortex. J Neurophysiol 84:1677-1680. 